\title{
Small- and Large-Stakes Risk Aversion: Implications of Concavity Calibration for Decision Theory*
}

\author{
James C. Cox ${ }^{\text {a,b }}$ and Vjollca Sadiraj ${ }^{\text {c,d }}$ \\ ${ }^{\mathrm{a}}$ Department of Economics, University of Arizona, Tucson, AZ 85721-0108, USA \\ ${ }^{\mathrm{b}}$ Workshop in Political Theory and Policy Analysis, Indiana University, Bloomington, IN \\ 47408-3895, USA \\ 'Economic Science Laboratory, University of Arizona, Tucson, AZ 85721-0108, USA \\ ${ }^{\mathrm{d}}$ CREED, University of Amsterdam, 1019 WB Amsterdam, The Netherlands
}

* This paper is a revised version of Cox and Sadiraj (2001). We are especially grateful to Martin Dufwenberg for constructive criticisms of earlier versions of this paper. Helpful comments and suggestions were provided by Glenn W. Harrison, Peter P. Wakker, and two anonymous referees. The National Science Foundation provided research support (grant numbers SES-9818561 and DUE-0226344).

Forthcoming in Games and Economic Behavior. 


\begin{abstract}
A growing literature reports the conclusions that: (a) expected utility theory does not provide a plausible theory of risk aversion for both small-stakes and large-stakes gambles; and (b) this decision theory should be replaced with an alternative theory characterized by loss aversion. This paper explains that the arguments in previous literature fail to support these conclusions. Either concavity calibration has no general implication for expected utility theory or it has problematic implications for all decision theories that involve concave transformations (utility or value functions) of positive money payoffs, which makes loss aversion irrelevant to the argument.
\end{abstract}

JEL classification: C90, D81

Keywords: concavity calibration, expected utility theory, prospect theory, risk aversion 


\section{Introduction}

Von Neumann and Morgenstern (1947) developed a theory of utility as an essential component of a theory of play for strategic games. Their utility theory, now known as expected utility theory, is based on a set of axioms for a preference ordering of probability distributions of "prizes.” The set of axioms includes the independence axiom which gives an expected utility functional representing the axioms its defining characteristic of linearity in probabilities. It was clearly understood in classic work (e.g., Luce and Raiffa, 1957, Ch. 2) that the axioms do not specify the identity of the prizes, such as scalar amounts of terminal wealth or income or, alternatively, commodity vectors. A difference in the assumed identity of the prizes is the characteristic that distinguishes one expected utility model from another. Failure in recent literature to distinguish between expected utility theory all models based on a set of axioms

that includes the independence axiom and a specific expected utility model has led to incorrect conclusions.

For example, Rabin and Thaler (2001, p. 221) building on earlier work by Rabin state that they “... establish the implausibility of expected utility theory by showing that absurd large-stakes risk aversion ... follow inherently from non-negligible modest-scale risk aversion ...." Validity of this Rabin-Thaler conclusion has been accepted in the academic literature (Kahneman, 2003; Camerer and Thaler, 2003) and general readership literature (The Economist, 2001) and in the award literature for the 2002 Nobel Prize in Economic Sciences (Royal Swedish Academy of Sciences, 2002, p. 16).

This paper explains that the concavity-calibration argument, as developed by Rabin (2000), does not logically support the implausibility conclusion about expected utility theory. Our 
explanation in section 2 begins with the original Rabin (2000) assumption that an agent will reject a 50-50 small-stakes gamble at all positive initial wealth levels. The section 2 explanation continues by providing: (a) an accessible demonstration that this small-stakes risk aversion assumption and globally-concave utility do imply implausible large-stakes risk aversion for the expected utility of terminal wealth model (see Rabin, 2000 for an original proof); and (b) a counterexample to illustrate that the small-stakes risk aversion assumption and global concavity do not imply implausible large-stakes risk aversion for the expected utility of income model. Since this small-stakes risk aversion assumption of Rabin (2000) does not imply implausible large-stakes risk aversion for the expected utility of income model, the conclusion by Rabin and Thaler (2001) that their arguments "establish the implausibility of expected utility theory" does not stand. Rubinstein (2001, 2004) presents a critique in a similar spirit.

The expected utility of income model is widely used in the theory of auctions. ${ }^{1}$ However, the model does not provide an explanation of how an agent's initial wealth affects its attitude towards risk. In order to be able to analyze the effects of initial wealth on risk-taking behavior, one needs a model in which risk attitude does depend on initial wealth. In order for a model to withstand the Rabin critique, initial wealth must not be additive to income in the utility function. This is our motivation for introducing, in section 3 , an expected utility model in which the arguments of the utility function are ordered pairs of initial wealth and income. To demonstrate that this new model, the expected utility of initial wealth and income model, may have fruitful applications we extend the Arrow (1971) and Pratt (1964) characterization of comparative risk aversion to it. The new expected utility model is not subject to Rabin's (2000) critique because his risk aversion assumption does not imply implausible large-stakes risk aversion for this model, as we illustrate with a counterexample.

Explaining that Rabin's (2000) small-stakes risk aversion assumption has no general implication for expected utility theory is the first topic addressed. Another topic addressed in this paper is exploring general implications of concavity calibration for decision theory. Many 
decision theories with "utility functionals" that are not linear in probabilities involve concave transformations (utility or value functions) of positive money payoffs, and therefore they may be vulnerable to an extended concavity calibration critique. Section 4 addresses this question. We present a more-general concavity calibration proposition that does not assume linearity in probabilities. Using this concavity calibration result, we identify an alternative small-stakes risk aversion assumption that implies that decision theories which involve concave transformations of positive money payoffs have implausible large-stakes risk aversion. Whether or not this pattern of small-stakes risk aversion has empirical validity is a question best addressed elsewhere, but the analysis of its implications demonstrates the following insights. The logic of concavity calibration has no special implication for expected utility theory. The implications for decision theory of the issues raised by Rabin are either much narrower or much broader than Rabin's (2000) and Rabin's and Thaler's (2001) conclusions suggest. Either concavity calibration has no general implication for expected utility theory (if only the Rabin calibration is considered), or there are

problematic implications for any decision theory that involves concave transformation of positive money payoffs (if other calibrations, such as ours in section 4, are considered). In the latter case, expected utility theory, cumulative prospect theory (Tversky and Kahneman, 1992) and other decision theories are subjected to a concavity-calibration critique. Furthermore, loss aversion is not a solution to the problems for decision theory that may follow from concavity calibration because our alternative small-stakes risk aversion assumption (in section 4) holds in the domain of positive money payoffs, which makes loss aversion irrelevant to the argument. ${ }^{2}$ This finding contradicts a conclusion by Rabin and Thaler (2001, p. 230) that loss aversion is a "key component” of a decision theory that can survive concavity calibration critique.

\section{Implications of the Rabin Risk Aversion Assumption for Expected Utility Theory}

In this section we re-examine the Rabin-Thaler (2001) conclusion, based on Rabin's (2000) calibration, that their arguments “...establish the implausibility of expected utility 
theory...” We provide straightforward demonstrations that Rabin's assumed pattern of smallstakes risk aversion: (a) does imply implausible large-stakes risk aversion for the expected utility of terminal wealth model; but (b) does not have an analogous implausible risk aversion implication for the expected utility of income model.

Rabin's (2000) analysis of the implications of small-stakes risk aversion begins with the assumption that an agent with (weakly) concave Bernoulli utility function will reject a smallstakes gamble with even odds of winning or losing relatively small amounts, and that the agent will do this at all positive initial wealth levels. ${ }^{3}$ We examine the implications of this assumption: (a) when the prizes are amounts of terminal wealth; and (b) when the prizes are amounts of income.

\subsection{Implications for the Expected Utility of Terminal Wealth Model}

Consider the expected utility of terminal wealth model, the model based on the expected utility axioms and the assumption that the prizes are amounts of terminal wealth. An accessible demonstration of the logic of concavity calibration for a differentiable utility function is as follows.

Assume that an agent rejects the gamble that involves a monetary gain of 110 and loss of 100, with even odds, for all values of initial wealth $w$ greater than $100 .{ }^{4}$ According to the expected utility of terminal wealth model, a necessary condition for rejecting the gamble is that the increase in utility by having 110 more is not larger than the decrease in utility by having 100 less, that is ${ }^{5}$

$$
u(w+110)-u(w) \leq u(w)-u(w-100), \text { for all } w>100 .
$$

We shall show that statement (1) and concavity of $u(\cdot)$ imply that an agent will reject any even odds gamble with a loss of at least 1,680 no matter how large is the gain amount. This 
demonstrates how a pattern of plausible small-stakes risk aversion implies implausible largestakes risk aversion for this model.

The driving mechanism of the concavity-calibration critique is that an implication of statement (1) and concavity of $u(\cdot)$ is geometrically diminishing marginal utility:

$$
u^{\prime}(w+210 t) \leq(100 / 110)^{t} u^{\prime}(w) \text {, for all } w>100 \text { and } t=1,2, \cdots
$$

In order to derive inequalities (2) first recall that, from the concavity of $u(\cdot)$, any straight line that connects two different points on the graph of the function has a weakly larger (smaller) slope than the tangent line at the largest (smallest) point. Hence, $\{[u(w+110)-u(w)] / 110\}$ $\geq u^{\prime}(w+110)$ and $\{[u(w)-u(w-100)] / 100\} \leq u^{\prime}(w-100)$. Therefore the term on the lefthand-side of inequality (1) is at least $110 u^{\prime}(w+110)$ whereas the term on the right-hand-side is at most $100 u^{\prime}(w-100)$. Thus, inequality (1) and concavity of $u(\cdot)$ imply that

$$
110 u^{\prime}(w+110) \leq 100 u^{\prime}(w-100) \text {, for all } w>100 \text {. }
$$

Inequality (3) shows that the marginal utility of getting $210(=w+110-(w-100))$ more money is not larger than $(100 / 110)$ times the marginal utility of the original amount. Since inequality (3) holds for all $w>100$, it holds for $w+210$ as well, and therefore the marginal utility at $(w+210)+210$ is at most $(100 / 110)$ of the marginal utility at $w+210$, which is at most $(100 / 110)$ of the marginal utility at $w$. Thus the marginal utility at $w+210 \times 2$ is at most $(100 / 110)^{2}$ of the marginal utility at $w$. Continuing the argument, one finds that for any given positive integer $t$, the marginal utility at $w+210 \times t$ is at most $(100 / 110)^{t}$ of the marginal utility at $w$, and therefore inequality (2) is shown to hold.

Implications of inequality (2) and global concavity of $u(\cdot)$ are as follows. From concavity, the increase in utility from having 210 more than some initial wealth $w$ is bounded from above by 210 times the marginal utility at $w$, i.e. $u(w+210)-u(w) \leq 210 u^{\prime}(w)$. 
Similarly, if initial wealth is $w+210$ then $u(w+210 \times 2)-u(w+210) \leq 210 u^{\prime}(w+210)$.

The two immediately-preceding inequalities together with inequality (2), written for $t=1$, imply the following inequality: $u(w+210 \times 2)-u(w) \leq 210[1+100 / 110] u^{\prime}(w)$. Hence, by iteration $u(w+210 \times J)-u(w) \leq 210\left[1+100 / 110+(100 / 110)^{2}+\cdots+(100 / 110)^{J-1}\right] u^{\prime}(w)$. Note that the preceding summation in brackets can never be larger than $11(=1 /(1-100 / 110))$. This result and positive monotonicity of $u(\cdot)$ imply: $\left(^{*}\right)$ the increase in utility from receiving any addition $G$ to initial wealth $w>100$ can never be larger than $[210 \times 11] u^{\prime}(w)$, which becomes $210 \times 11$ if we normalize the utility function so that $u^{\prime}(w)=1$. On the other hand, for any positive integer $k$ such that $w-210 k>100$, writing inequality (2) for wealth equal to $w-210 k$ and $t=1$ yields $u^{\prime}((w-210 k)+210) \leq(100 / 110) u^{\prime}(w-210 k)$. Hence the marginal utility at $w-210 k$ is at least $(110 / 100)$ of the marginal utility at $w-210 k+210(=w-210(k-1))$. By repeating this argument, one finds that the marginal utility at $w-210 k$ is at least $(110 / 100)^{k}$ of the marginal utility at $w$. Following the same logic as above, and for $u^{\prime}(w)=1$, one observes that the utility of $210 \times 1$ less than $w$ is at least 210 less than the utility of $w$; the utility of $210 \times 2$ less than $w$ is at least $210(1+110 / 100)$ less than the utility of $w$, and by iteration the utility of $210 \times K$ less than $w$ is at least $210\left[1+110 / 100+\cdots+(110 / 100)^{K-1}\right]$ less than the utility of $w$. The summation in brackets is strictly larger than 11 for all positive integers $K>7$; therefore $u(w)-u(w-210 K)>210 \times 11$, for all such $K$. This result and positive monotonicity of $u(\cdot)$ imply: $(* *)$ the decrease in utility from any subtraction $L \geq 1,680(=8 \times 210)$ from initial wealth $w>L$ is larger than $210 \times 11$. Statements $\left(^{*}\right)$ and $\left({ }^{* *}\right)$ and transitivity imply that the increase in utility from a gain of any amount $G$, no matter how large, is strictly smaller than the decrease in utility from a loss of 1,680 (or any larger loss) at any $w$ bigger than the loss amount. This is a sufficient condition for rejection of any even-odds gamble, no matter how large the gain, that 
involves a loss of 1,680 (or more). Therefore, the assumed pattern of plausible small-stakes risk aversion implies implausible large-stakes risk aversion for the expected utility of terminal wealth model.

\subsection{Implications for the Expected Utility of Income Model}

Now consider the expected utility of income model, the model based on the expected utility axioms and the assumption that the prizes are amounts of income (or changes in wealth). We shall demonstrate that the Rabin (2000) small-stakes risk aversion assumption does not imply implausible large-stakes risk aversion for this model.

Let $\mu$ denote the agent's Bernoulli utility function for income. A necessary condition for rejecting an even-odds gamble with loss amount $\ell$ and gain amount $g$ at all wealth levels $w>\ell$ is:

$$
\mu(g)-\mu(0) \leq \mu(0)-\mu(-\ell) .
$$

It should be expected that the Rabin concavity calibration has no large-stakes risk aversion implications for this model because inequality (4) does not depend on $w$. In order to explicate comparison with the terminal wealth model, we consider the same small-stakes gamble as in section 2.1: receive -100 or +110 with even odds. An example of a Bernoulli utility function $\mu$ for income $y$ that rejects the gamble with outcomes -100 or +110 with even odds, for all $w>100$, and has plausible large-stakes risk aversion is:

$$
\begin{aligned}
\mu(y) & =0.9 y+0.1, \text { for } y<1, \\
= & y^{0.9}, \text { otherwise. }
\end{aligned}
$$

Using utility function (5), define $g(\ell)$ as the amount of gain that makes the agent indifferent between accepting and rejecting the even-odds gamble with loss amount $\ell$, that is $g(\ell)$ satisfies weak inequality (4) with an equality. Straightforwardly, if $\ell>1$ then 
$g(\ell)=(0.1+0.9 \ell)^{10 / 9}$ as a solution to the equation $(g(\ell))^{0.9}-0.1=0.1-(0.9 \times(-\ell)+0.1)$.

An even odds gamble with loss amount $\ell$ and gain amount $G$ will be rejected (accepted) for all gain amounts $G$ strictly smaller (larger) than $g(\ell)$. Hence, the even-odds lottery with outcomes of -100 and +110 is rejected since the gain amount 110 is strictly smaller than $g(100) \approx 149$. However, in contrast to the terminal wealth model, the agent with utility of income function (5) would accept an even-odds gamble with loss 1,680 and gain at least 3,412 since that is strictly larger than $g(1,680) \approx 3,411 .{ }^{6}$ Thus, the assumed pattern of risk aversion over small-stakes gambles does not imply implausible risk aversion over large-stakes gambles with this expected utility model.

\section{Expected Utility of Initial Wealth and Income Model}

The expected utility of income model is widely used in the theory of auctions but this model does not provide an explanation of how an agent's initial wealth affects its attitude towards risk. In this section we consider a model in which risk attitude depends on initial wealth but income is not additive to initial wealth.

Assume that the arguments of the utility function are ordered pairs of initial wealth and income. Let $v$ denote the agent's "Bernoulli” utility function for initial wealth and income. For any integrable probability distribution function $G$ for random income $y$, the expected utility functional for this model is written as

$$
\int v(w, y) d G=E_{G}(v(w, y))
$$

where the function $v$ is strictly increasing in both arguments and (resp. strictly) concave in its second argument if the agent is (resp. strictly) risk averse. Although, in this model, risk attitude depends on initial wealth, the model is not called into question by the type of global small-stakes risk aversion assumed in previous literature, as we shall now demonstrate. 


\subsection{Rationalizing Small- and Large-Stakes Risk Aversion}

For the model of initial wealth and income, a necessary condition for rejecting an evenodds gamble with loss amount $\ell$ and gain amount $g$ for all positive $w$ is

$$
v(w, g)-v(w, 0) \leq v(w, 0)-v(w,-\ell), \text { for all } w>0
$$

We present an example of a "Bernoulli" function utility $v$ that both satisfies inequality (7) for small-stakes risky lotteries and has plausible large-stakes risk aversion implications. To keep things comparable among the three expected utility models, we consider the same small-stakes gamble as in sections 2.1 and 2.2, the even-odds gamble with outcomes -100 and +110 .

Consider the following "Bernoulli" utility function $v$ for initial wealth $w$ and income $y:{ }^{7}$

$$
\begin{aligned}
v(w, y) & =\frac{\left(0.9 y+0.1-e^{-w}\right)}{\left(1-e^{-w}\right)^{0.1}}, \quad y<1, \\
& =\left(y-e^{-w}\right)^{0.9}, \text { otherwise. }
\end{aligned}
$$

This utility function satisfies the pattern of risk aversion assumed by Rabin (2000) and at the same time it has plausible large-stakes risk aversion, as shown below.

For the utility function (8), the amount of gain $g(\ell, w)$ that makes the agent indifferent between accepting and rejecting the even-odds gamble with loss amount $\ell>1$ is given by $g(\ell, w)=e^{-w}+\left[\left(0.9 \ell+0.1-e^{-w}\right) /\left(1-e^{-w}\right)^{0.1}\right]^{10 / 9}$, which is derived by solving weak inequality (7) with equality. An even-odds gamble with outcomes $-\ell$ and $+g$ will be rejected (accepted) for $g$ strictly smaller (larger) than $g(\ell, w)$. Note that for initial wealth $w>100$ and loss amount $\ell=100$, one has $g(100, w) \approx 149$. Therefore $110<g(100, w)$ for all $w>100$, which implies rejection of the even-odds gamble with outcomes -100 and +110 . However, in contrast to the terminal wealth model's prediction, the agent would accept an even-odds gamble 
with loss 1,680 and gain at least 3,412 since $g(1680, w) \approx 3,411$ for all $w>0$. Thus the assumed pattern of risk aversion over small-stakes gambles does not imply implausible risk aversion over large-stakes gambles with the expected utility of initial wealth and income model.

\subsection{Comparative Risk Aversion}

Having observed that the expected utility of initial wealth and income model is not called into question by the type of global small-stakes risk aversion assumed in previous literature, the next question is whether this model can be used in applications in which the central questions are concerned with the implications of different attitudes towards risk and their possible dependence on initial wealth. We address this question by extending the Arrow-Pratt characterization of comparative risk aversion to the new model.

The "Bernoulli" utility functions for two agents can be written as $v^{j}(w, y)$, for $j=\alpha, \beta$. The measure of absolute risk aversion for this model is

$$
A^{j}(w, y)=-\frac{v_{22}^{j}(w, y)}{v_{2}^{j}(w, y)}
$$

Let $\bar{y}$ be the mean value of income for the distribution $G$; then the risk premium, $\pi^{j}$ is defined by

$$
v^{j}\left(w, \bar{y}-\pi^{j}(w, G)\right)=E_{G}\left(v^{j}(w, y)\right) .
$$

Given that the function $v^{j}$ is strictly increasing in its second argument, $y$ there exists a $y$ inverse function $\phi^{j}$ defined by

$$
y=\phi^{j}\left(w, v^{j}(w, y)\right) .
$$

Define the function $g$ as follows:

$$
g(w, u)=v^{\alpha}\left(w, \phi^{\beta}(w, u)\right) .
$$


The measures of comparative risk attitudes for agents $\alpha$ and $\beta$ are as given in the following proposition, which states that: (i) the absolute risk aversion measure for agent $\alpha$ is greater than the absolute risk aversion measure for agent $\beta$, if and only if, (ii) the risk premium for agent $\alpha$ is greater than the risk premium for agent $\beta$, if and only if, (iii) the utility function for agent $\alpha$ is a strictly increasing and strictly concave transformation of the utility function of agent $\beta$ of the form given by the definition in equation (12).

Proposition 1. If $v^{\alpha}$ and $v^{\beta}$ are strictly increasing in $y$ and twice differentiable then the following statements are equivalent:

(i) $A^{\alpha}(w, y)>A^{\beta}(w, y)$, for all $(w, y)$;

(ii) $\pi^{\alpha}(w, G)>\pi^{\beta}(w, G)$, for all $w$ and $G$;

(iii) $v^{\alpha}(w, y)=g\left(w, v^{\beta}(w, y)\right), g_{2}(w, u)>0, g_{22}(w, u)<0$, for all $(w, u)$.

Proof: See appendix B.

Proposition 1 makes clear that the Arrow-Pratt characterization of agents' comparative risk aversion can be extended from the expected utility of terminal wealth model to the twoargument, expected utility of initial wealth and income model which is not called into question by Rabin's (2000) concavity-calibration arguments. Hence, rather than using the expected utility of income model, agents’ risk-avoiding behavior can be modeled with the new model. ${ }^{8}$

\section{More Implications of Concavity Calibration for Decision Theory}

In sections 2 and 3, we demonstrated that the Rabin (2000) small-stakes risk aversion assumption has implausible large-stakes risk aversion implications for only one of the three expected utility models that we examined. This was the first question addressed in this paper. This section addresses another question: What are the possible implications of concavity 
calibration for decision-theoretic models other than the expected utility of terminal wealth model? We present a concavity calibration proposition for functionals that represent preferences on a lottery space that may not be linear in probabilities (as are expected utility functionals). This more general concavity calibration proposition identifies small-stakes risk aversion assumptions for which all decision theories that involve concave transformations of positive money payoffs have implausible large-stakes risk aversion. This alternative pattern of small-stakes risk aversion may or may not have empirical validity, but the analysis makes it clear that the logic of concavity calibration has no unique implication for expected utility theory; either concavity calibration has no general implication for expected utility theory (because it does not apply to the expected utility of income model nor to the expected utility of initial wealth and income model), or it has problematic implications for all decision theories that involve concave transformations of positive money payoffs.

\subsection{Implications for Decision Theory of an Alternative Pattern of Risk Aversion}

Consider binary gambles that pay the amount of income $x$ with probability $p$ and the amount of income $y$ with probability $1-p$, where $p \in[0,1]$. Denote such a gamble by $\{p ; x, y\}$. Consider a decision theory $D$ that represents a preference ordering of these binary gambles with a functional $F_{D}=(h, f)$ given as

$$
F_{D}(p ; x, y)=h(p) f(x)+(1-h(p)) f(y) .
$$

That is, according to decision theory $D$, lottery $\gamma=\{p ; a, b\}$ is preferred to lottery $\delta=\{q ; c, d\}$ if and only if $F_{D}(\gamma)>F_{D}(\delta)$, which using (13) can be written as

$$
\gamma \succ \delta \text { iff } h(p) f(a)+(1-h(p)) f(b)>h(q) f(c)+(1-h(q)) f(d) .
$$

For a functional representation $F_{D}=(h, f)$, function $h$ is commonly called a probability transformation function and function $f$ is called a money transformation (or utility or value) 
function. In the case of expected utility theory, the probability transformation function is the identity map: $h(p)=p$, for all $p \in[0,1]$.

We define a gamble $\{p ; x-\ell, x+g\}$ to be $D$-favorable if it satisfies

$$
h(p)(x-\ell)+(1-h(p))(x+g)>x .
$$

Then one has the following proposition. ${ }^{9}$

Proposition 2. Let an agent's preference ordering be represented according to some decision theory $D$ by a functional $F_{D}=(h, f)$. Let $0<\ell<g$ be given and $f$ be increasing, concave and differentiable for positive amounts of money. Suppose that the agent prefers a certain positive amount of money $x$ to a D-favorable lottery $\{p ; x-\ell, x+g\}$ for all $x \in(\ell,+\infty)$. Then according to decision theory $D$ there exists a finite positive $L^{*}$ such that for all $L>L^{*}$ and $z>L$ the agent will prefer the certain positive amount of money $z$ to lottery $\{p ; z-L, G\}$, for all $G$.

Proof: See appendix C, part C.1.

Interpretation of the definition of $D$-favorable lottery in inequality (15) for the special case of expected utility theory is that the gamble has positive expected value. In order to further explicate which lotteries are $D$-favorable, note that a risky lottery $\{p ; x-\ell, x+g\}$ is $D$-favorable if and only if $g \times(1-h(p))-\ell \times h(p)>0$. Since the last inequality does not depend on $x$, one has: if $\left\{p ; x^{*}-\ell, x^{*}+g\right\}$ is $D$-favorable for some $x^{*} \in(\ell,+\infty)$ then $\{p ; x-\ell, x+g\}$ is $D$-favorable for all $x \in(\ell,+\infty)$.

\subsection{Interpretations with Alternative Decision Theories}


We here offer some interpretations of Proposition 2 for models from two alternative decision theories, expected utility theory and cumulative prospect theory. First consider the three expected utility models discussed in sections 2 and 3 of this paper, the terminal wealth model, the income model, and the initial wealth and income model. Let the preference ordering over lotteries according to any of these three models be represented by the functional $E=(i d, U)$, where id denotes the identity map which is the probability transformation for expected utility theory. The concave money payoff transformation function $U$ is the Bernoulli utility function for income $\mu(x)$, or terminal wealth $u(w+x)$, or initial wealth and income $v(w, x)$, depending on which model is considered. Binary lottery $\{0.5 ; x-100, x+110\}$ is an $E$-favorable risky gamble. For all three expected utility models discussed above, applying Proposition 2 and using the constructive proof in appendix C, part C.2, one has: rejection of this risky gamble in favor of a certain amount of income $x$, for all $x>100$, implies rejection of any even-odds lottery that involves a lower outcome less than $z-1,680$ in favor of receiving a certain amount $z(z>1,680)$, no matter how large is the high outcome. An example is: 6,000 for sure is preferred to an even-odds lottery with low outcome of 4,000 and any arbitrarily-large high outcome. Thus we have the following corollary.

Corollary 1. Any of the three expected utility models predict: if $x \succ\{0.5 ; x-100, x+110\}$, $\forall x>100$, then $\forall L \geq 1,680$ and $\forall z>L, z \succ\{0.5 ; z-L, G\}$ for all $G$.

Note that the examples of utility functions given in equations (5) and (8) do not satisfy the antecedent statement in Corollary 1 and, therefore, the corollary does not apply to those preferences.

Next consider cumulative prospect theory (Tversky and Kahneman, 1992). Let $P=(\mathrm{W}, v)$ denote the functional that represents the preference ordering according to this theory. Since all 
outcomes are positive, the relevant money transformation function is the concave value function for gains and the relevant probability transformation function is given by the probability weighting function for gains $W^{+}$, with $W^{+}(0.5)=0.58$ (Tversky and Kahneman, 1992, p. 312). First, consider the gamble $\{0.5 ; x-75, x+110\}$. This gamble is $P$-favorable, as defined in statement (15), because $0.58(x-75)+0.42(x+110)>x$. Applying Proposition 2 for decision theory $P$ (see appendix C, part C.3), one gets: if the certain amount of money $x$ is preferred to $\{0.5 ; x-75, x+110\}$, for all $x>75$, then for all $L>1,850$ and $z>L$, the certain amount of money $z$ is preferred to $\{0.5 ; z-L, G\}$ no matter how large is $G .{ }^{10}$ The example reported for the expected utility models can be used here as well. That is, the derivation in part C.3 of appendix C implies that any even-odds gamble with lower outcome of 4,000, no matter how large the other outcome may be, is rejected in favor of receiving 6,000 for sure. Alternatively, consider the gamble $\{0.36 ; x-100, x+110\}$. Gonzales and Wu (1999, p. 157) report on persistence across studies of the estimate $W^{+}(0.36)=0.5$, and therefore the gamble $\{0.36 ; x-100, x+110\}$ is $P$ favorable as defined in statement (15). Applying Proposition 2, one finds that rejection of gamble $\{0.36 ; x-100, x+110\}$ in favor of a certain amount of money $x$, for all $x>100$, implies rejection of $\{0.36 ; z-L, G\}, \forall G>0$ in favor of receiving $z$ for sure, for all $L>1,680$ and $z>L$ (see appendix C, part C.3). Again, the example reported above survives (in a more extreme form): the gamble of receiving 4,000 with probability 0.36 or any positive amount with probability 0.64 is rejected in favor of receiving 6,000 for sure. The following corollary summarizes these findings.

Corollary 2. Cumulative prospect theory $P$ with the probability transformation function $W$, as reported in the literature, predicts:

$$
\text { if } x \succ\{0.5 ; x-75, x+110\} \text {, for all } x>75 \text {, then } \forall L \geq 1,850 \text { and } \forall z>L
$$




$$
z \succ\{0.5 ; z-L, G\} \text {, for all } G \text {; }
$$

$$
\begin{aligned}
& \text { if } x \succ\{0.36 ; x-100, x+110\} \text {, for all } x>100 \text {, then } \forall L \geq 1,680 \text { and } \forall z>L \\
& z \succ\{0.36 ; z-L, G\} \text {, for all } G \text {. }
\end{aligned}
$$

Therefore, the presently-assumed global small-stakes risk aversion and concavity of the value function for positive amounts of money imply implausible large-stakes risk aversion with cumulative prospect theory.

The assumption in Proposition 2 that an agent prefers the certain amount of money $x$ to a $D$-favorable lottery $\{p ; x-\ell, x+g\}$, for all $g>\ell$ and $x>\ell>0$, implies that the money transformation (utility or value) function $f$ is bounded (see appendix C, part C.4). ${ }^{11}$ However, the implication of implausible large-stakes risk aversion in Proposition 2 and its corollaries does not require bounded money transformation functions. Implausible large-stakes risk aversion is also implied by an assumption that a certain amount of money $x$ is preferred to a $D$-favorable lottery $\{p ; x-\ell, x+g\}$, for all $x$ in a sufficiently large finite interval, and this alternative assumption does not imply bounded money transformation functions. (A proof of this alternative version of Proposition 2 is available upon request to the authors.)

\section{Concluding Remarks}

This paper explains that the type of global small-stakes risk aversion assumed in previous literature (Rabin, 2000; Rabin and Thaler, 2001) has no implication for the expected utility of income model, hence no general implication for expected utility theory. However, an agent’s risk attitude with the income model does not depend on initial wealth. This is our motivation for discussion of a two-argument model for which risk attitude does depend on initial wealth. We show that this model is immune to the concavity-calibration critique in previous literature. An 
extension of the Arrow-Pratt characterization of comparative risk aversion to this new model is presented, demonstrating that this two-argument model may have fruitful applications.

In order to explore the implications of concavity calibration for decision theory, we present a concavity calibration proposition that does not assume linearity in probabilities. This proposition, together with an alternative pattern of global small-stakes risk aversion, provides a concavity-calibration critique that applies to all expected utility models reported in this paper, to cumulative prospect theory, and to other decision theories that involve concave money transformation functions for positive money payoffs. ${ }^{12}$ The alternative assumed pattern of risk aversion holds in the domain of gains, which makes loss aversion irrelevant to the argument. This makes it clear that loss aversion does not provide a way around any problems for decision theory that follow from concavity calibration. This new pattern of small-stakes risk aversion, as assumed in Proposition 2, may or may not have empirical validity; the central role of Proposition 2 in the analysis is to make clear that concavity calibration has no unique implication for expected utility theory. ${ }^{13}$ Instead, concavity calibration either has no general implication for expected utility theory or it has problematic implications for all decision theories with concave transformation (utility or value) functions for positive money payoffs. 


\section{Endnotes}

1. Vickrey (1961) first developed Nash equilibrium bidding theory based on the expected utility axioms. He mainly developed the theory for the special case of risk neutral bidders, for which there is no essential distinction between the expected utility of terminal wealth model and the expected utility of income model. Later authors used the expected utility of income model to develop Nash equilibrium bidding theory for risk averse agents. See, for examples: Holt (1980), Harris and Raviv (1981), Riley and Samuelson (1981), Cox, Smith, and Walker (1982), Milgrom and Weber (1982), Matthews (1983), Maskin and Riley (1984), and Moore (1984). The bid functions for risk averse bidders presented in these papers do not satisfy the best reply property of Nash equilibrium if one assumes that the argument of the utility function is terminal wealth rather than income. Appendix A provides an illustration.

2. Loss aversion can be incorporated into a decision-theoretic model by assuming a utility or value function for income that has a kink at zero income and is steeper in the loss domain than in the gain domain (Kahneman and Tversky, 1979). While it has been featured in discussions of prospect theory, loss aversion is also consistent with the expected utility of income model and the new expected utility model introduced in this paper.

3. Rabin (2000) examines the implications of rejection of this small-stakes gamble for all $w \in I$, where $I$ is either an infinite or sufficiently large finite interval. We focus on the infinite interval in order to simplify the exposition of concavity calibration.

4. The wealth levels at which the small-stakes gamble is assumed to be rejected are required to be greater than the loss amount in the gamble (here, 100) because negative terminal wealth is not well-defined. 
5. According to the expected utility of terminal wealth model, a necessary condition for rejecting gambles with outcomes $x$ and $-y$ at even-odds is $0.5 u(w+x)+0.5 u(w-y) \leq u(w)$, which is equivalent to $u(w+x)-u(w) \leq u(w)-u(w-y)$. Strict inequality gives a sufficient condition. We use this characterization for rejection (or acceptance for the opposite inequality sign) of evenodds lotteries for the rest of the paper.

6. Examples of other gambles that would be accepted include: $\{0.5 ;-2000,+4150\}$; $\{0.5 ;-10000,+24760\}$; and $\{0.5 ;-20000,+53470\}$.

7. This function is risk neutral for income less than 1 , otherwise it exhibits absolute risk aversion (as defined in statement (9)) that is decreasing in both income and wealth.

8. Note that, as presently developed this model shares some limitations with the expected utility of income model, prospect theory, and other models defined on income: it does not rule out certain types of anomalies (see Rubinstein (2004) for an illustration). Detailed analysis of possible "money pump” preference cycles and other violations of full rationality are beyond the scope of the present paper, which is concerned with the implications of concavity calibration for decision theories.

9. Proposition 2 holds for non-differentiable $f$ as well. Furthermore, implausible large-stakes risk aversion is implied even if small-stakes risk aversion is assumed to hold in a (large enough) finite interval instead of the infinite one. An alternative proposition (and proof) that relaxes differentiability of $f$ and the infinite interval assumption is available upon request to the authors.

10. The lower outcome $z-L$ is required to be positive in order to make loss aversion irrelevant to the argument.

11. We thank Martin Dufwenberg for bringing this to our attention. 
12. An example of a decision theory that does not involve concave transformation of money payoffs is provided by Yaari’s (1987) dual theory of choice under risk. The "utility functional" that represents the dual theory preference ordering is always linear in money payoffs but is linear in probabilities only if the agent is risk neutral.

13. The empirical credibility of Rabin's (2000) risk aversion assumption is disputed in PalaciosHuerta, Serrano, and Volij (2003) and in Cox and Sadiraj (2001). In ongoing empirical research, we are attempting to obtain data that can shed light on the empirical validity of Rabin's assumption and the alternative risk aversion assumption in Proposition 2. 


\section{References}

Arrow, K., 1971. Essays in the Theory of Risk-Bearing. Markham Publishing Co, Chicago.

Camerer, C., Thaler, R.H., 2003. In Honor of Matthew Rabin: Winner of the John Bates Clark Medal. Journal of Economic Perspectives 17, 159-176.

Cox, J. C., Sadiraj, V., 2001. Risk Aversion and Expected Utility Theory: Coherence for Smalland Large-Stakes Gambles. Working Paper 01-03, Department of Economics, University of Arizona.

Cox, J. C., Smith, V. L., Walker, J. M., 1982. Auction Market Theory of Heterogeneous Bidders. Economics Letters 9, 319-325.

Gonzales, R., Wu, G., 1999. On the Shape of the Probability Weighting Function. Cognitive Psychology 38, 129-166.

Harris, M., Raviv, A., 1981. Allocation Mechanisms and the Design of Auctions. Econometrica 49, 1477-1499.

Holt, C. A., Jr., 1980. Competitive Bidding for Contracts Under Alternative Auction Procedures. Journal of Political Economy 88, 433-445.

Kahneman, D., 2003. A Psychological Perspective on Economics. American Economic Review Papers and Proceedings 93, 162-168.

Kahneman, D., Tversky, A., 1979. Prospect Theory: An Analysis of Decision under Risk. Econometrica 47, 263-292.

Luce, R. D., Raiffa, H., 1957. Games and Decisions. Wiley, New York.

Maskin, E., Riley, R., 1984. Optimal Auctions with Risk Averse Buyers. Econometrica 52, 14731518.

Matthews, S. A., 1983. Selling to Risk Averse Buyers with Unobservable Tastes. Journal of Economic Theory 30, 370-400.

Milgrom, P. R., Weber, R. J., 1982. A Theory of Auctions and Competitive Bidding. Econometrica 50, 1089-1122.

Moore, J., 1984. Global Incentive Constraints in Auction Design. Econometrica 52, 1523-1536.

von Neumann, J., Morgenstern, O., 1947. Theory of Games and Economic Behavior. Princeton University Press, Princeton NJ.

Palacios-Huerta, I., Serrano, R., Volij, O., 2003. Rejecting Small Gambles under Expected Utility. Working paper, Brown University.

Pratt, J. W. , 1964. Risk Aversion in the Small and in the Large. Econometrica 32, 122-136. 
Rabin, M., 2000. Risk Aversion and Expected Utility Theory: A Calibration Theorem. Econometrica 68, 1281-1292.

Rabin, M., Thaler, R. H., 2001. Anomalies: Risk Aversion. Journal of Economic Perspectives 15, 219-232.

Riley, J. G., Samuelson, W. F., 1981. Optimal Auctions. American Economic Review 71, 381392.

Royal Swedish Academy of Sciences, 2002. Foundations of Behavioral and Experimental Economics: Daniel Kahneman and Vernon Smith. Advanced Information on the Prize in Economic Sciences 17, 1-25.

Rubinstein, A., 2001. Comments on the Risk and Time Preferences in Economics. Working paper, Tel Aviv University.

Rubinstein, A., 2004. Dilemmas of an Economic Theorist. Presidential Address, Econometric Society.

The Economist, 2001. Economics focus: Averse to reality. August $11^{\text {th }}, 61$.

Tversky, A., Kahneman, D., 1992. Advances in Prospect Theory: Cumulative Representation of Uncertainty. Journal of Risk and Uncertainty 5, 297-323.

Yaari, M. E., 1987. The Dual Theory of Choice under Risk. Econometrica 55, 95-115.

Vickrey, W., 1961. Counterspeculation, Auctions, and Competitive Sealed Tenders. Journal of Finance 16, 8-37. 


\section{Appendix A. Bidding Theory for Risk Averse Agents Works with the Expected Utility of Income Model}

In order to appreciate that the terminal wealth model is not used in the literature on bidding theory, consider a special case example from the theory of first-price sealed-bid auctions of single items with independent private values (Cox, Smith, and Walker, 1982). Let private values be drawn from the uniform distribution on [0,1]. Let the preferences of 2 bidders be represented by the Bernoulli utility function for income $y: u(y)=y^{r}$ for $r \in(0,1)$. Then the Nash equilibrium bid function is: $(*) b_{i}=v_{i} /(1+r)$. Now consider the terminal wealth model instead. For this model the best reply property of Nash equilibrium is violated. Indeed, since the $v$-inverse of bid function $(*)$ is $v_{i}=(1+r) b_{i}$, the probability that bidder 1 will bid less than $b_{2}$ is $(1+r) b_{2}$, given that bidder 1 bids according to bid function $(*)$. Using the terminal wealth model, the expected utility of bidder 2 from bidding amount $b_{2}$ is $U\left(b_{2}\right)=(1+r) b_{2}\left(w+v_{2}-b_{2}\right)^{r}+\left[1-(1+r) b_{2}\right] w^{r}$. It can be easily verified that $b_{2}=v_{2} /(1+r)$ does not satisfy the first order condition for a maximum of $U\left(b_{2}\right)$ given that $0<r<1$. Furthermore, replacing $v_{i}$ by $w_{i}+v_{i}$ in bid function $(*)$ does not yield a bid function with the mutual best reply property, nor does replacing $v_{i}$ by $w+v_{i}$ for the special case $w_{1}=w_{2}=w$. Examples using other Nash equilibrium bid functions in the literature lead to the same conclusion that the required best reply property is not satisfied if one uses the expected utility of terminal wealth model rather than the expected utility of income model. 


\section{Appendix B. Proof of Proposition 1}

Note that $v^{\alpha}(w, y)=g\left(w, v^{\beta}(w, y)\right)$ is true by construction (see statements (11) and

(12)). Furthermore, $g_{2}(w, u)>0, \forall(w, u)$, because $v_{2}^{\alpha}=g_{2} v_{2}^{\beta}$, and $v_{2}^{\alpha}(w, y)>0$ and $v_{2}^{\beta}(w, y)>0, \forall(w, y)$

We first show that statements (i) and (iii) in Proposition 1 imply each other: (i) $\leftrightarrow$ (iii). Differentiation of the functions in statement (iii) with respect to $y$ yields

$$
\begin{aligned}
& v_{2}^{\alpha}=g_{2} v_{2}^{\beta} \text {, and } \\
& v_{22}^{\alpha}=g_{22}\left(v_{2}^{\beta}\right)^{2}+g_{2} v_{22}^{\beta} .
\end{aligned}
$$

The definition of $A^{j}(w, y)$ and statements (b.1) and (b.2) imply

$$
g_{22}=\frac{v_{22}^{\alpha}-g_{2} v_{22}^{\beta}}{\left(v_{2}^{\beta}\right)^{2}}=\left[\frac{v_{22}^{\alpha}}{v_{2}^{\alpha}}-\frac{v_{22}^{\beta}}{v_{2}^{\beta}}\right] \frac{v_{2}^{\alpha}}{\left(v_{2}^{\beta}\right)^{2}}=\left[\mathrm{A}^{\beta}-A^{\alpha}\right] \frac{v_{2}^{\alpha}}{\left(v_{2}^{\beta}\right)^{2}} .
$$

Statement (b.3) implies that $g_{22}(w, u)<0, \forall(w, u)$, if and only if $A^{\alpha}(w, y)>A^{\beta}(w, y), \forall(w, y)$.

We next show that statement (iii) in Proposition 1 implies statement (ii) in the proposition: (iii) $\rightarrow$ (ii). Jensen’s inequality and the definitions imply

$$
\begin{aligned}
& v^{\alpha}\left(w, \phi^{\beta}\left(w, E_{G}\left(v^{\beta}(w, y)\right)\right)\right)=g\left(w, E_{G}\left(v^{\beta}(w, y)\right)\right) \\
& >E\left(g\left(w, v^{\beta}(w, y)\right)\right)=E\left(v^{\alpha}\left(w, \phi^{\beta}\left(w, v^{\beta}(w, y)\right)\right)\right)=E\left(v^{\alpha}(w, y)\right) .
\end{aligned}
$$

Therefore

$$
\begin{aligned}
& E_{G}(y)-\pi^{\beta}(w, G)=\phi^{\beta}\left(w, E_{G}\left(v^{\beta}(w, y)\right)\right)=\phi^{\alpha}\left(w, v^{\alpha}\left(w, \phi^{\beta}\left(w, E_{G}\left(v^{\beta}(w, y)\right)\right)\right)\right) \\
& >\phi^{\alpha}\left(w, E_{G}\left(v^{\alpha}(w, y)\right)\right)=E_{G}(y)-\pi^{\alpha}(w, G) .
\end{aligned}
$$

Therefore statement (iii) implies statement (ii) in Proposition 1.

We next show that statement (ii) in Proposition 1 implies statement (iii) in the proposition: (ii) $\rightarrow$ (iii). Statement (ii) and the definitions imply 


$$
\begin{aligned}
& v^{\alpha}\left(w, \phi^{\beta}\left(w, E_{G}\left(v^{\beta}(w, y)\right)\right)\right)=v^{\alpha}\left(w, E_{G}(y)-\pi^{\beta}(w, G)\right) \\
& >v^{\alpha}\left(w, E_{G}(y)-\pi^{\alpha}(w, G)\right)=E_{G}\left(v^{\alpha}(w, y)\right) .
\end{aligned}
$$

Hence

$$
\begin{aligned}
& g\left(w, E_{G}\left(v^{\beta}(w, y)\right)\right)=v^{\alpha}\left(w, \phi^{\beta}\left(w, E_{G}\left(v^{\beta}(w, y)\right)\right)\right) \\
& >E_{G}\left(v^{\alpha}(w, y)\right)=E_{G}\left(v^{\alpha}\left(w, \phi^{\beta}\left(w, v^{\beta}(w, y)\right)\right)\right)=E_{G}\left(g\left(w, v^{\beta}(w, y)\right)\right) .
\end{aligned}
$$

Therefore, $g$ is strictly concave in $u: g_{22}(w, u)<0, \forall(w, u)$. 


\section{Appendix C. Proof of Proposition 2 and Corollaries 1 and 2}

\section{C.1 Proof of Proposition 2}

Let a decision theory $D$ with functional $F_{D}=(h, f)$ be given. Let money transformation function $f$ have the following properties for positive amounts of money: increasing, concave and differentiable. Let $0<\ell<g$ be given. Suppose that the agent prefers a positive amount of money $x$ for sure to a $D$-favorable lottery, $\{p ; x-\ell, x+g\}$ for all $x \in(\ell,+\infty)$. This assumption and statement (14) in the text for decision theory $D$ imply

$$
f(x) \geq h(p) f(x-\ell)+(1-h(p)) f(x+g) \text {, for all } x>\ell .
$$

We shall show that (c.1) and the concavity property of $f$ imply that for all positive $z$ and positive integers $j$,

$$
f^{\prime}(z+j(\ell+g)) \leq\left(\frac{h(p)}{1-h(p)} \frac{\ell}{g}\right)^{j} f^{\prime}(z)
$$

Note that $h(p) \neq 1$ since $\{p ; x-\ell, x+g\}$ is a $D$-favorable lottery.

We shall also show that there exists a $K^{*}$ such that for all positive integers $K>K^{*}$, for all $z>(\ell+g) K$

$$
h(p)[f(z)-f(z-(\ell+g) K)]>(1-h(p))[f(z+(\ell+g) J)-f(z)],
$$

for all positive integers $J$. This will give us our result because statement (14) and inequality (c.3) imply that for all $K>K^{*}$, and for all $z>(\ell+g) K$, the agent prefers the positive amount of money $z$ for sure to the lottery $\{p ; z-(\ell+g) K, z+(\ell+g) J\}$ for all $J$. This preference for $z$, together with positive monotonicity of $f$, implies Proposition 2 with $L^{*}=(\ell+g) K^{*}$.

To derive (c.2), note that inequality (c.1) holds for all $x>\ell$, and thus it holds for $z+\ell$

$$
f(z+\ell) \geq h(p) f(z)+(1-h(p)) f(z+\ell+g) \text {, for all positive } z \text {. }
$$

Substituting $f(z+\ell)=h(p) f(z+\ell)+(1-h(p)) f(z+\ell)$ in (c.4) and rewriting it, one has 
(c.5) $\quad h(p)[f(z+\ell)-f(z)] \geq[1-h(p)][f(z+\ell+g)-f(z+\ell)]$, for all positive $z$.

Inequalities $[f(z+\ell+g)-f(z+\ell)] / g \geq f^{\prime}(z+\ell+g)$ and $[f(z+\ell)-f(z)] / \ell \leq f^{\prime}(z)$, (both following from the concavity property of $f$ ) and inequality (c.5) imply

$$
f^{\prime}(z+(\ell+g)) \leq\left(\frac{h(p)}{1-h(p)} \frac{\ell}{g}\right) f^{\prime}(z) \text {, for all positive } z
$$

Iterative application of the logic used to derive (c.6) implies

$$
f^{\prime}(z+j(\ell+g)) \leq\left(\frac{h(p)}{1-h(p)} \frac{\ell}{g}\right) f^{\prime}(z+(j-1)(\ell+g)) \leq \ldots \leq\left(\frac{h(p)}{1-h(p)} \frac{\ell}{g}\right)^{j} f^{\prime}(z), \text { which }
$$

gives statement (c.2).

To derive statement (c.3) note that for all positive integers $J$, and for all positive $Z$, one has

$$
\begin{aligned}
f(z+(\ell+g) J)-f(z) & =\sum_{j=0}^{J-1}[f(z+(j+1)(\ell+g))-f(z+j(\ell+g))] \\
& \leq(\ell+g) \sum_{j=0}^{J-1} f^{\prime}(z+j(\ell+g)) \\
& \leq(\ell+g) f^{\prime}(z) \sum_{j=0}^{J-1}\left(\frac{h(p)}{1-h(p)} \times \frac{\ell}{g}\right)^{j},
\end{aligned}
$$

where the first inequality follows from the concavity property of $f$ and the second one follows from statement (c.2). Similarly, for any given positive integer $K$ and for all $Z$ larger than $(\ell+g) K$

$$
f(z)-f(z-(\ell+g) K) \geq(\ell+g) f^{\prime}(z) \sum_{k=0}^{K-1}\left(\frac{1-h(p)}{h(p)} \times \frac{g}{\ell}\right)^{k}
$$

Note that $h(p) \neq 0$ follows from inequality (c.1) and $f$ being an increasing function. Showing that there exists a positive $K^{*}$ such that for all positive integers $K>K^{*}$, 


$$
(1-h(p)) \sum_{j=0}^{J-1}\left(\frac{h(p)}{1-h(p)} \times \frac{\ell}{g}\right)^{j}<h(p) \sum_{k=0}^{K-1}\left(\frac{1-h(p)}{h(p)} \times \frac{g}{\ell}\right)^{k}, \text { for all } J \geq 1 \text {, }
$$

completes the proof since then (c.7), (c.8) and (c.9) imply (c.3). Inequality (c.9) is a direct implication of the binary lottery $\{p ; x-\ell, x+g\}$ being a $D$-favorable one. Indeed, statement (15) in the text implies $(h(p) /(1-h(p))) \times(\ell / g)<1$, and hence for all values of $J$ the term on the left-hand-side of (c.9) is strictly smaller than the finite number $S$ defined as $S \equiv(1-h(p)) \sum_{j=0}^{\infty}\left(\frac{h(p)}{1-h(p)} \times \frac{\ell}{g}\right)^{j}=(1-h(p)) /\left(1-\frac{h(p)}{1-h(p)} \times \frac{\ell}{g}\right)$; whereas the term on the

right-hand-side of inequality (c.9) is unbounded from above as $K \rightarrow \infty$ (since $((1-h(p)) / h(p)) \times(g / \ell)>1)$. Therefore, there exists a positive integer $K^{*}$ that makes the right-hand-side term in (c.9) larger than $S$, and hence strictly larger than the left-hand-side term for all $J$.

\section{C.2. Proof of Corollary 1}

For $\ell=100, g=110, p=0.5$ and $h(0.5)=0.5$, one has $S=5.5$, and the right hand side of inequality (с.9) is larger than 5.5 for all $K \geq K^{*}=8$. Hence, Proposition 2 applies with $L^{*}=(110+100) \times 8$.

\section{C.3. Proof of Corollary 2.}

Part (i). For this case, $\ell=75, g=110, p=0.5$ and $h(0.5)=W^{+}(0.5)=0.58$ and hence $S \approx 7.2$, and the right hand side of inequality (с.9) becomes larger than 7.2 for all $K \geq K^{*}=10$. Then, apply Proposition 2 with $L^{*}=(110+75) \times 10$. 
Part (ii). For this case, $\ell=100, g=110, p=0.36$ and $h(0.36)=W^{+}(0.35)=0.5$, and one can verify that $S=5.5$ and that the right hand side of inequality (c.9) is larger than 5.5 for all $K \geq K^{*}=8$. Then apply Proposition 2 with $L^{*}=210 \times 8$.

\section{C.4. Note about Bounded Money Transformation Functions}

Assumption (c.1) and concavity imply that function $f$ is bounded from above as follows. For any given $x$, there exists a positive integer $J$ such that $x \leq(1+(\ell+g) J)$, and therefore $f(x) \leq f(1+(\ell+g) J)$. The right-hand-side of the last inequality is smaller than $\left[f(1)+(\ell+g) f^{\prime}(1) S /(1-h(p))\right]$ which follows from inequality (c.7) and the definition of $S$. Denoting the last expression by $\mathrm{M}$, one has $f(x) \leq M$ for all $x$. 